\title{
Enhanced cognitive state (ECS)
}

Jonathan D. Nash, Andrew Newberg, Bhuvanesh Awasthi

\section{Source}

Jonathan D. Nash, Andrew Newberg. (2013). Toward a unifying taxonomy and definition

formeditation. Front. Psychol., vol. 4 . doi:10.3389/fpsyg.2013.00806.

Is defined as the resultant state of consciousness due to the successful employment of a $C D M$, in which the phenomenological content is primarily cog nitive in nature. 